\title{
Auditory and visual systems organization in Brodmann Area 8 for gaze-shift control: where we do not see, we can hear
}

\author{
Marco Lanzilotto ${ }^{1,2 *}$, Vincenzo Perciavalle ${ }^{3}$ and Cristina Lucchetti ${ }^{1,2}$ \\ ${ }^{1}$ Section of Physiology and Neuroscience, Department of Biomedical Sciences, Metabolic and Neuroscience, University of Modena and Reggio Emilia, Modena, \\ Italy \\ ${ }^{2}$ Section of Polyclinic, Interdepartmental Facilities Center, University of Modena and Reggio Emilia, Modena, Italy \\ ${ }^{3}$ Section of Physiology, Department of Biomedical Sciences, University of Catania, Catania, Italy \\ *Correspondence: marco.lanzilotto@unimore.it
}

Edited by:

Katharina A. Braun, Otto-von-Guericke University, Germany

Reviewed by:

Sophie Molholms, Albert Einstein College of Medicine, USA

Keywords: FEF, PEEF, auditory system, visual system, oculomotor system, gaze shift, monkey

Hearing is especially important for most primate species as they live in habitats of dense vegetation that limits vision. Stebbins (1980) summed up the evolution of the auditory system by assuming that earliest mammals exploited nocturnal niches since they were relatively free of many of the large, diurnal, predacious reptiles. Therefore, hearing and smell were more useful at night than vision.

Our vision is limited not only in the dark but also outside the visual field. In fact, if we observe the behavior of a predator like a feline, oriented toward its prey, and at the same time a sound occurs behind, we might note three principal different behaviors: the predator could maintain its gaze and ears on the prey neglecting the sound source; the predator could maintain its gaze on the prey rotating ears and then shifting its auditory attention toward the sound source; finally the predator could break its attention and orient gaze and ears toward the sound source. A similar behavior is seen in human beings during social interaction with two or more interlocutors.

In humans, orienting movements are carried out by the eyes, head, and/or body operating alone or in various combinations depending on the behavioral situation. However, in non-human primates, such as macaque monkeys, head orienting movements and, more generally, gaze-shift are accompanied by ear orienting movements, which allow the shifting of auditory attention toward a sound of interest (Bon and Lucchetti, 1994, 2006; Lucchetti et al., 2008; Lanzilotto et al., 2013; Yin, 2013).
Considering all these assumptions, the auditory system could have an important role to detect information even from regions of the space that the visual system cannot explore without orienting movements. In other words, where we cannot see, we can hear.

Through this opinion article, we argue that Brodmann Area 8 receives information from both auditory and visual systems and organizes a transformation of these sensory signals into gaze-shift motor commands. Our hypothesis is that this sensory-motor transformation is spatially organized, from both anatomical and functional points of view.

Anatomical and functional properties of the Brodmann Area 8 (consisting in Area 8A plus Area 8B) support a mediolateral organization for both auditory and visual systems. In particular, the lateral portion, corresponding to Area 8A or Frontal Eye Field (FEF), could play a role in receiving visual and auditory information from a central part of the visual field and then in organizing gaze-shift motor commands toward it. Otherwise, the medial portion, corresponding to Area $8 \mathrm{~B}$ or Premotor Ear-Eye Field (PEEF), could play a role in receiving principally auditory information from a peripheral region of the space and then in organizing gaze-shift motor commands toward it.

\section{FRONTAL EYE FIELD}

The (FEF, area $8 \mathrm{~A}$ ) is a functional field in the prefrontal cortex, located in the rostral bank of the arcuate sulcus of macaques. FEF participates in the transformation of visual signals into saccade motor commands (Schall, 1997; Sato et al., 2001). FEF is innervated in a topographic fashion by areas of both the dorsal and ventral visual streams originating in extrastriate visual cortex (Schall et al., 1995). The ventral part of the FEF receives visual information from areas where fovea is clearly represented and retinotopically organized, such as MT and V4; from areas in the inferotemporal cortex such as TEO and caudal TE involved in central vision; finally from areas in parietal cortex having poor retinotopic organization but important for oculomotor transformation, such as LIP. In support of anatomical evidence, Suzuki and Azuma (1983) found that neurons located in the ventral FEF had relatively small visual receptive fields (RFs) representing the foveal and parafoveal regions. Moreover the ventral FEF works for generating short amplitude saccades (Bruce et al., 1985). On the contrary, the dorsal part of FEF is connected with retinotopically organized areas where peripheral visual field is represented, such as areas MSTd and PO, located medially, that are involved in peripheral vision, as well as from LIP. In support of anatomical evidence, Suzuki and Azuma (1983) found that neurons located in dorsal FEF had larger RFs and eccentric from the fovea. Finally, the dorsal FEF is responsible for generating larger amplitude saccades (Bruce et al., 1985).

Otherwise, it is well known, that FEF receive also information from the auditory system, participating thus, in the transformation of auditory signals into motor 


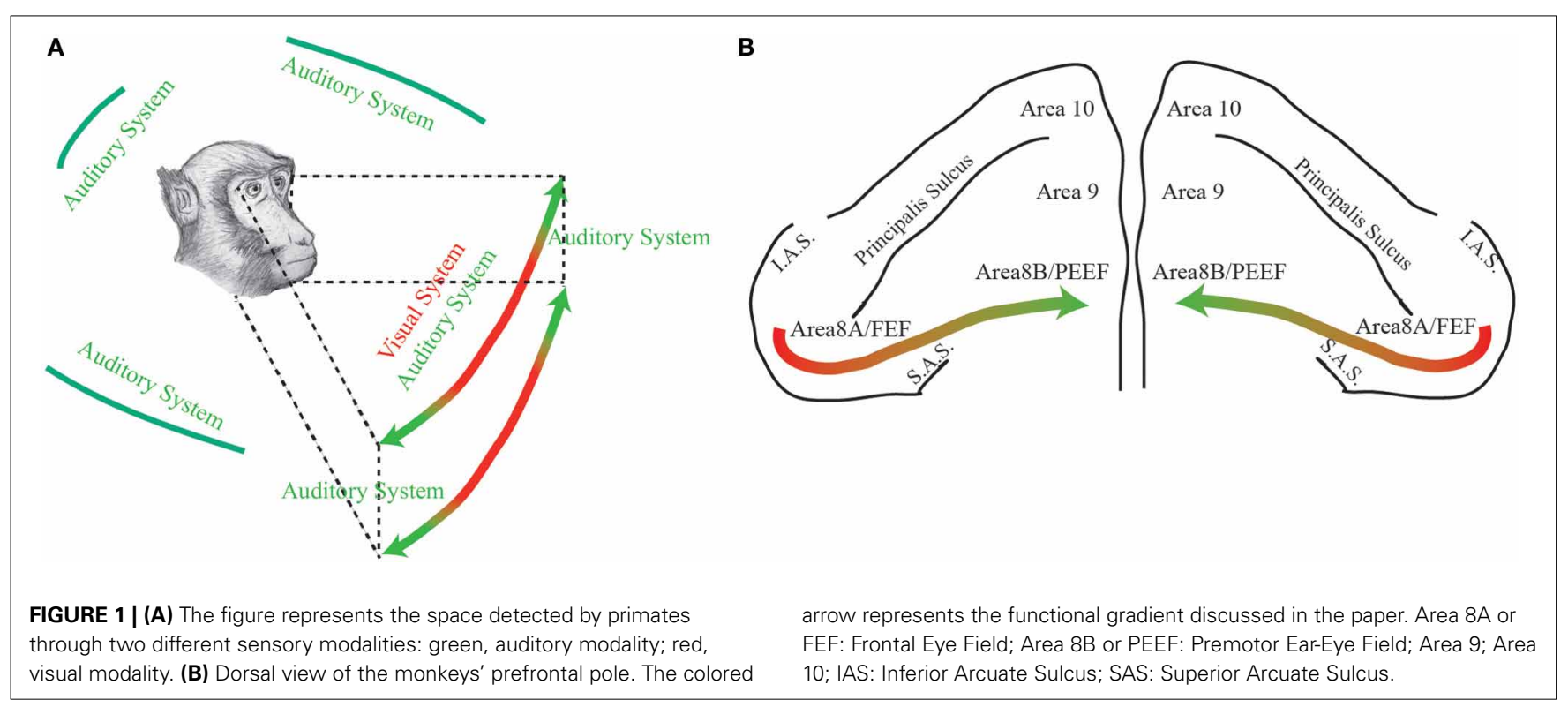

commands. An interesting study show in fact as FEF, in human, is a multimodal area involved in ultra-rapid responses for auditory and visual stimuli (Kirchner et al., 2009).

The auditory cortical system is constituted by two different streams, termed dorsal and ventral, projecting to the frontal cortex in non-human primates (Romanski et al., 1999a,b). The dorsal stream directly projects from caudal auditory belt to area 8A (Romanski et al., 1999a), bringing information about sound spatial localization. The ventral stream, involved in codifying the features of the auditory stimulus, originating from rostral auditory belt and rostral auditory parabelt, is connected indirectly to area $8 \mathrm{~A}$ passing through the ventral prefrontal cortex (Romanski et al., 1999a; Gerbella et al., 2010; Rauschecker and Romanski, 2011).

In accord to anatomical evidence, many neurons in FEF respond to auditory stimuli. FEF has an interesting medio-lateral gradient regarding both the auditory spatial selectivity and proportion of auditory neurons. In particular, Azuma and Suzuki (1984) showed that in in the ventral part of FEF, auditory neurons have RFs located at $10^{\circ}$ respect to the azimuth. As one moves more medially toward dorsal FEF, auditory neurons have more eccentric RFs till $40^{\circ}$ respect to the azimuth. Moreover, in dorsal FEF the number of auditory neurons gradually increases while the number of visual neurons gradually decreases.

\section{PREMOTOR EAR EYE FIELD}

Recently, area 8B, located medially to FEF, has been renamed as a new frontal field: PEEF (Lucchetti et al., 2008; Bon et al., 2009; Lanzilotto et al., 2013). PEEF is characterized by having principally connections with the auditory system, and has been hypothesized to participate in the transformation of auditory signals into motor commands. In particular, PEEF is connected directly to the caudal auditory belt through the dorsal auditory stream (Romanski et al., 1999a), which has a role for sound spatial localization. In accord to these connections, three principal classes of neurons have been identified in PEEF: auditory neurons, auditory-motor neurons, and motor neurons. The auditory stimuli that better elicit a response in PEEF's neurons are complex environmental stimuli as experimenter voices, while ear and/or eye movements represent the motor effectors controlled by this region. For these reasons, PEEF has been proposed as a new field having a possible role to detect auditory stimuli in the space through ear and eye movements (Lanzilotto et al., 2013).

\section{CONCLUSION}

Through this opinion article, in light of PEEF discovery (Lucchetti et al., 2008; Bon et al., 2009; Lanzilotto et al., 2013), we propose that the part of the frontal cortical region, consisting of area $8 \mathrm{~A}$ (FEF) plus area $8 \mathrm{~B}$ (PEEF), could be thought having a medio-lateral organization devoted for the exploration of different regions of the space. In particular, the lateral partwhich receives visual information from retinotopically organized areas and has auditory neurons with RFs close to the azimuth-could have an important role in receiving visual and auditory information from a central region of the space and then in organizing eye motor commands toward it. On the other hand, the medial part-which receives, principally, auditory information through the dorsal auditory stream-could have an important role in receiving auditory information from a peripheral region of the space and then in organizing ear-eye motor commands toward it (Figure 1).

\section{ACKNOWLEDGMENTS}

This work was supported by Department of Biomedical Sciences, University of Catania ("Progetti di Ricerca di Ateneo 2011"). We thank Prof. Gianfranco Franchi for his comments.

\section{REFERENCES}

Azuma, M., and Suzuki, H. (1984). Properties and distribution of auditory neurons in the dorsolateral prefrontal cortex of the alert monkey. Brain Res. 298, 343-346. doi: 10.1016/0006-8993(84)91434-3

Bon, L., Lanzilotto, M., and Lucchetti, C. (2009). "PEEF: premotor ear-eye field. A new vista of area 8B," in Prefrontal Cortex: Roles, Interventions and Traumas, eds L. LoGrasso and G. Morretti (Portland, OR: Nova Science Publisher), 157-175.

Bon, L., and Lucchetti, C. (1994). Ear and eye representation in the frontal cortex, area $8 \mathrm{~b}$, of 
the macaque monkey: an electrophysiological study. Exp. Brain Res. 102, 259-271. doi: 10.1007/BF00227513

Bon, L., and Lucchetti, C. (2006). Auditory environmental cells and visual fixation effect in area $8 \mathrm{~B}$ of macaque monkey. Exp. Brain Res. 168, 441-449. doi: 10.1007/s00221-005-0197-5

Bruce, C. J., Goldberg, M. E., Bushnell, M. C., and Stanton, G. B. (1985). Primate frontal eye fields. II. Physiological and anatomical correlates of electrically evoked eye movements. J. Neurophysiol. 54, 714-734.

Gerbella, M., Belmalih, A., Borra, E., Rozzi, S., and Luppino, G. (2010). Cortical connections of the macaque caudal ventrolateral prefrontal areas $45 \mathrm{~A}$ and 45B. Cereb. Cortex 20, 141-168. doi: 10.1093/ cercor/bhp087

Kirchner, H., Barbeau, E. J., Thorpe, S. J., Regis, J., and Liegeois-Chauvel, C. (2009). Ultra-rapid sensory responses in the human frontal eye field region. J. Neurosci. 29, 7599-7606. doi: 10.1523/ JNEUROSCI.1233-09.2009

Lanzilotto, M., Perciavalle, V., and Lucchetti, C. (2013). A new field in monkey's frontal cortex: premotor ear-eye field (PEEF). Neurosci. Biobehav. Rev. 29, 1434-1444. doi: 10.1016/j.neubiorev.2013. 05.010

Lucchetti, C., Lanzilotto, M., and Bon, L. (2008). Auditory-motor and cognitive aspects in area $8 \mathrm{~B}$ of macaque monkey's frontal cortex: a premotor ear-eye field (PEEF). Exp. Brain Res. 186, 131-141. doi: 10.1007/s00221-007-1216-5
Rauschecker, J. P., and Romanski, L. M. (2011). "Auditory cortical organization: evidence for functional streams," in The Auditory Cortex, eds. J. A. Winer and C. E. Schreiner (New York, NY: Springer), 99-116.

Romanski, L. M., Bates, J. F., and Goldman-Rakic, P. S. (1999a). Auditory belt and parabelt projections to the prefrontal cortex in the Rhesus monkey. J. Comp. Neurol. 403, 141-157. doi: 10.1002/ (SICI) 1096-9861(19990111)403:2<141::AID-CN $\mathrm{E} 1>3.0 . \mathrm{CO} ; 2-\mathrm{V}$

Romanski, L. M., Tian, B., Fritz, J., Mishkin, M., Goldman-Rakic, P. S., and Rauschecker, J. P. (1999b). Dual streams of auditory afferents target multiple domains in the primate prefrontal cortex. Nat. Neurosci. 2, 1131-1136. doi: 10.1038/ 16056

Sato, T., Murthy, A., Thompson, K. G., and Schall, J. D. (2001). Search efficiency but not response interference affects visual selection in frontal eye field. Neuron 30, 583-591. doi: 10.1016/S08966273(01)00304-X

Schall, J. D. (ed.). (1997). Visuomotor Areas of the Frontal Lobe. New York, NY: Plenum Press.

Schall, J. D., Morel, A., King, D. J., and Bullier, J. (1995). Topography of visual cortex connections with frontal eye field in macaque: convergence and segregation of processing streams. J. Neurosci. 15, 4464-4487.

Stebbins, W. (1980). "The evolution of hearing in the mammals," in Comparative Studies of Hearing in
Vertebrates, eds A. Popper and R. Fay (New York, NY: Springer), 421-436. doi: 10.1007/978-1-46138074-0_15

Suzuki, H., and Azuma, M. (1983). Topographic studies on visual neurons in the dorsolateral prefrontal cortex of the monkey. Exp. Brain Res. 53, 47-58. doi: 10.1007/ BF00239397

Yin, T. C. (2013). Physiological and behavioral studies of sound localization. J. Acoust. Soc. Am. 133, 3331. doi: $10.1121 / 1.4805588$

Received: 01 October 2013; accepted: 24 November 2013; published online: 10 December 2013.

Citation: Lanzilotto M, Perciavalle $V$ and Lucchetti $C$ (2013) Auditory and visual systems organization in Brodmann Area 8 for gaze-shift control: where we do not see, we can hear. Front. Behav. Neurosci. 7:198. doi: 10.3389/fnbeh.2013.00198

This article was submitted to the journal Frontiers in Behavioral Neuroscience.

Copyright (C) 2013 Lanzilotto, Perciavalle and Lucchetti. This is an open-access article distributed under the terms of the Creative Commons Attribution License (CC BY). The use, distribution or reproduction in other forums is permitted, provided the original author(s) or licensor are credited and that the original publication in this journal is cited, in accordance with accepted academic practice. No use, distribution or reproduction is permitted which does not comply with these terms. 